\title{
Automatic Classification of Autism using Fusion of EEG and 4D FMRI
}

\author{
K.Thaiyalnayaki
}

\begin{abstract}
The identification and classification of diseased networks in fMRI is very difficult mastermind in people with big running autism has demonstrated to have diminished integration beyond field of renewal regulated by fMRI. When looking at the resting essential structure of people with independent and rule individuals coordinated for dotage and knowledge result, the outcome demonstrate that those pairs have a quiet essential structure that is fundamentally same as together in amount and in constitution, but in solitary this grid is extra unfined related. The exact forecast of general neuropsychiatric issues, on an individual basis, using rs-fMRI is a challenging task of incredible clinical noteworthiness. By developing a system which process and classify the fMRI data, it can be easily predicted whether the neuropsychiatric disorders especially autism is present or not. The fusion of features of EEG signal and the features obtained from independent component of fMRI are utilized for the automatic classification of Autism disorder. It can also be used to identify the diseased network and to automatically classify the different components of diseased networks. A classifier is constructed by $k$-means on a $2 D$ feature projection space, with groupwise normalization for the classification of HC and Autism subjects with EEG and Rs-fMRI $4 D$ dataset and compared with Convolution Neural network $(C N N)$.
\end{abstract}

Keywords : fmRI, EEG,2D projection space.

\section{INTRODUCTION}

\section{Functional Magnetic Resonance Imaging}

fMRI is a strategy for contemplating the subjective operatin of the brain. The innovation of present day brain scaning methods, similar as positron emission Tomography (PET) \& fMRI, furnish another set of tools with which to study cerebral functional classifications. Autism spectrum disorder (ASD) is the flag for a body of enhancing anarchy. Citizens with ASD regularly have these characteristics, continuous issues that incorporate trouble imparting \& associating with others, tedious practices just as restricted interests or activities, demonstration that discomfort the human retention to perform culturally, at society everyday issues. The symptoms and signs include getting steamed at a slight change in a daily schedule or being upset in Parents, being strong visual and auditory learners.

The brain regions/areas responsible for the autism spectrum behavior are Broadmann area 17, primary visual cortex also known as Striate layer. The optical layer of the brain is a part of the deep layer that assumes a significant

Revised Manuscript Received on December 11, 2019

* Correspondence Author

K.Thaiyalnayaki *, Associate Professor, Department of ECE, Saveetha School of Engineering, SIMATS, Chennai. job in handling visual data. Optical data coming from eye experiences oblique crooked core, detected in the neural structure and arrives at the optical core. Also in Broadmann area 37(posterior inferior temporal gyrus, middle temporal gyrus and fusiform gyrus), the subordinate temporal gyrus is put beneath the middle temporal gyrus, and is associated behind with the inferior occipital gyrus, it likewise stretches out over the infero-lateral boundary on inferior surface of the temperol lobe. Broadmann area 20 that includes the inferior temporal, fusiform and parahippocampal gyri. The parahippocampal place zone is a sub region of the parahippocampal cortex that recline medially in the inferior temporo-occipital cortex. fMRI studies show that this area of the brain turns out to be profoundly dynamic when human subjects view topographical scene stimuli, for example, pictures of scenes, cityscapes, or rooms, pictures of spots. The Broadmann area 22, Superior temporal gyrus is situated somewhat above the external ear, the cortical region responsible for the sensation of sound. Peripheral nervous system of ASD, brain happening in cells stay obscure and determination based on observable situation. Developing documentation recommends that intellect abnormality in ASD happen at the stage of conjoined grid however utilizing operational integration information for checking classification have arrived at just balanced precision[1]. A matrix of practical networks between 220 functionally characterized field of activity is valued for checking, constructing few machines acquirement contraption, while holds heading instruments performed modestly, diagnostic classification arrived at a high accuracy of $91 \%$. For next stage the IC fingerprints naturally neglect the algorithm called machine learning and it splits into six stages after basic training. [2].

$\mathrm{CNN}$ is utilized in the group Senile psychosis's mind against typical sound intellect. The significance of variation sort of therapeutic information is to possibly build up a envision structure or framework so as to perceive the sort of disorder from typical gauge the phase of the disorder [3]. A new methodology for distinguishing significant contrasts in smart exercises between neurotypical subjects and Autism Spectrum Disorder (ASD) patients using the rs-fMRI. This performance got utilizing the log-variance features is seen as better when contrasted with earlier studies in the literature.[4] Elaheh Moradia explains the syndrome austerity based on compactness dimension from 156 substance with (ASD) for encounter structural brain malfunction in ASD multiprotocol[5].

Published By:

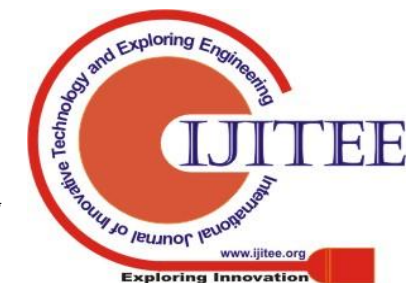


Investigations of fMRI have revealed insight to comprehension of the aspect of peripheral systems in ache handling and ache observation. GLM avoids to exploit the connection of fMRI data at various areas. SVM [12] (Support Vector Machine) classification is used for fMRI data to discover deep activations. [6] In Network based analysis they explored the Structural brain network contrasts in youngsters and youths with ADHD and healthy controls [7]. The advantages of this technique are using Network Based Statistic (NBS), ADHD had a sub network of stronger connectivity. By inspecting different methodological approaches, appears to deliver most stability and robust network contrasts across t- threshold [8-9]. The disadvantage of this technique is contributor gesture can have a consequence, regardless of no group dissimilarity in motion being perceived [10-11]. The constructively recognize such network deformity resting state functional MRI (RS-fMRI).

\section{PROPOSED WORK}

\section{Data Acquisition}

The EEG and fMRI data required for the analysis is obtained in parallel from both the design paradigm. The 4D fMRI as shown in figure 2.1 is acquired for rest or gaze state.

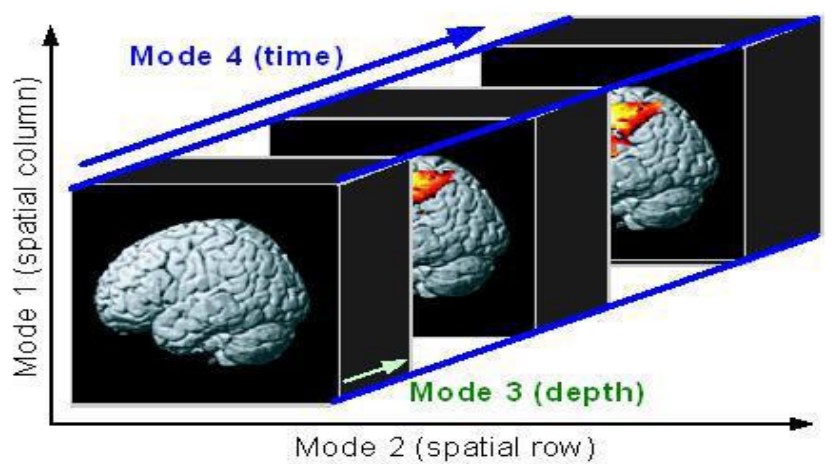

Fig 2.1: 4-D fMRI data

Good The fMRI scanner produces a diagrammatic representation of area being scanned which is represented as voxels. The fMRI voxels typically represent a Volume of 27 $\mathrm{mm} 3$. The various pre-processing steps in SPM are: Realignment, Normalization, Smoothing. First the realignment is to compensate for the movement of the head between scans, typically the images will be changed so that each of the voxels in the images corresponds approximately to the same brain site. This is called rearrangement or correction of motion. Two stage of realignments are estimate movement and resample images based on estimated movement. The normalization is ultimate goal of spatial normalization, which is the spatial coordination and conversion of the brain into a common space, making them equivalent to each other and the first scan. SPM will then write structured, spatially to the functional information directory. Prefix $w$ is assigned to these files. The smoothing is where images are often smoothed by voxels that are averaged with their neighbours. Spatial smoothing is intended to deal with functional anatomic variation that is not compensated by spatial normalization and enhance SNR.
Resting state fMRI is attained on a Siemens Tim Trio 3-Tesla scanner. The acquired fMRI data after preprocessing using SPM is applied through ICA. To identify spatially or temporally independent component from fMRI datasets, Group ICA technique is used. Networks were extracted via group independent component analysis (gICA).Few regions obtained using ICA is shown in Fig. 3.2. From the 70 independent components obtained, the features such as kurtosis, mean, median, variance, standard deviation are obtained. SVM classifier as shown in Fig. 3.1 is constructed to identify the autistic fMRI from the healthy controls. Comparing with the existing systems, the processes involved in this system are very simple because it classifies the data into only two classes namely autistic and non-autistic. So this process can be used to detect whether the neuropsychiatric disorders is present or not and the classification accuracy of the designed algorithm depends on the number of samples accurately classified, true positives t 0 true negatives. The classification accuracy reaches $81.1 \%$ on par with the existing classification algorithms.

\section{RESULT AND DISCUSSION}

After acquisition of EEG and fMRI of autism and healthy controls, the database is created and communicated to server for authentication and security. A data Repository is created in cloud where encryption and provision for backup of data are provided as shown in Fig.3.3. Stored EEG signals are preprocessed by passing through a $40 \mathrm{~Hz}$ LPF to remove the high frequency noise, the EEG signals may come across. Five BPF are constructed to separate alpha, beta, gamma, delta, theta signals. The obtained signals are transformed to wavelet domain and transform features are collected.

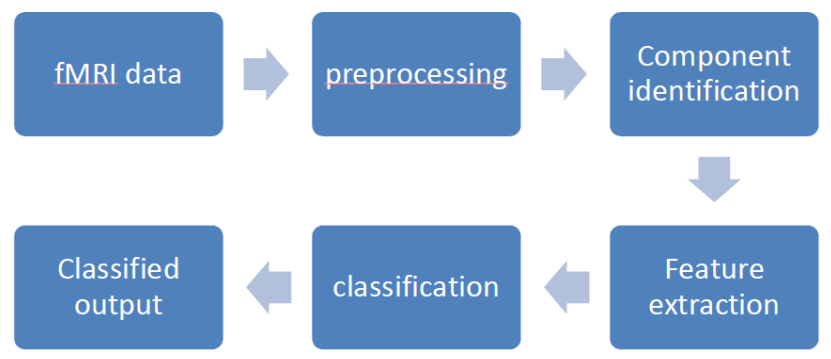

Fig.3.1 fMRI autism SVM Classifier without fusion

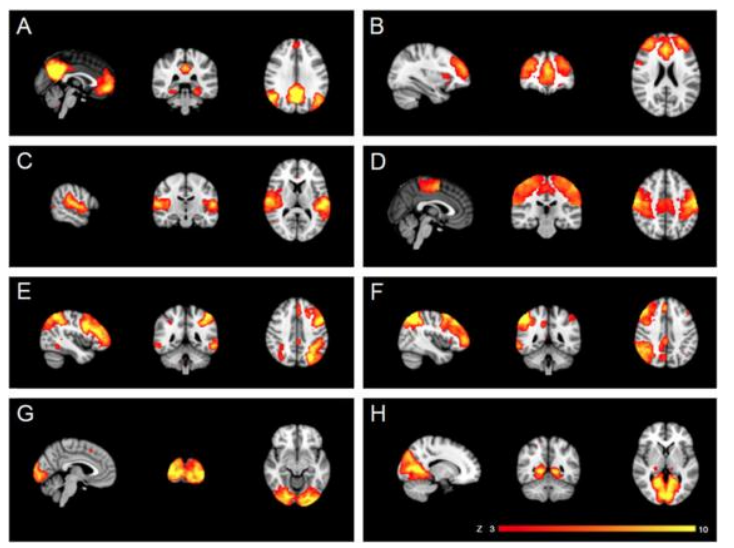

Fig. 3.2 Eight regions of Independent component analysis 


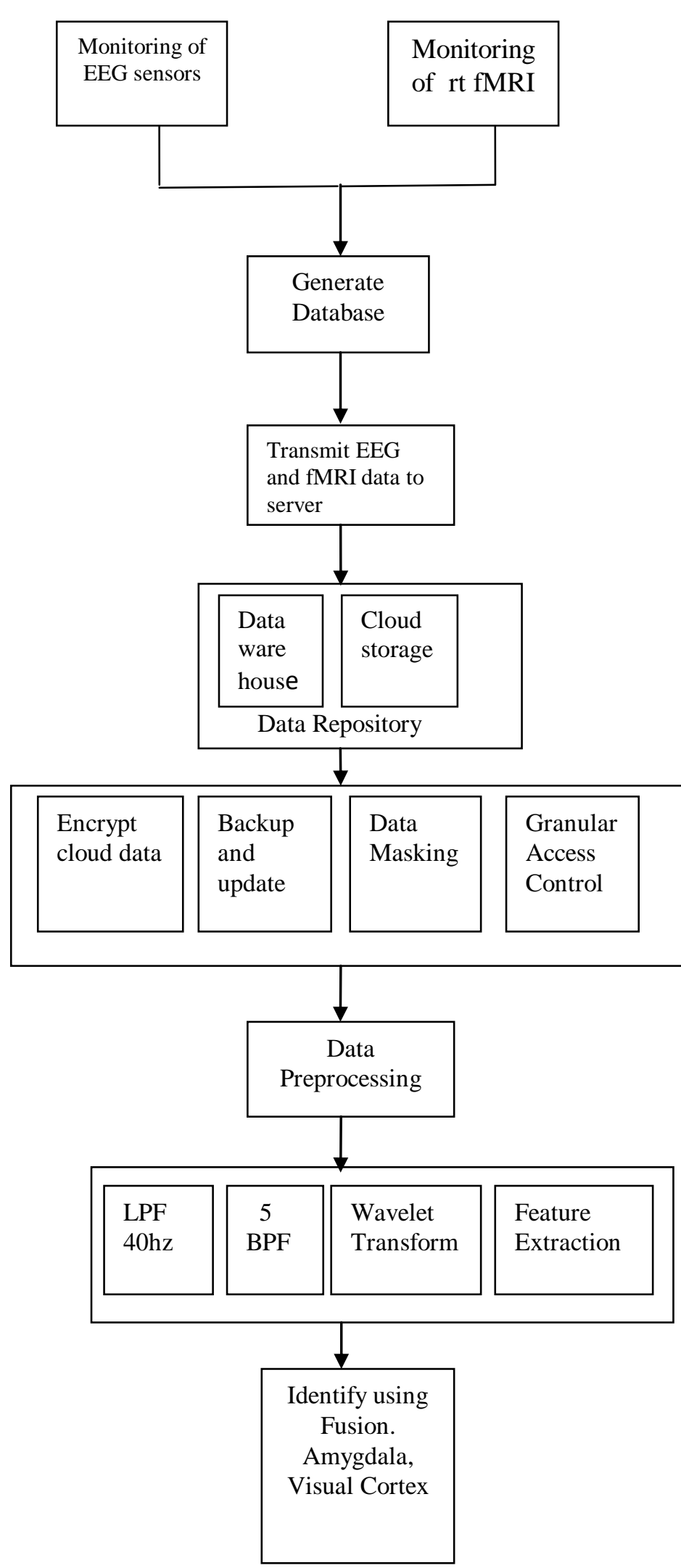

Fig.3.3 fMRI autism Classification using fusion

The features obtained from EEG and fMRI are combined to classify the healthy controls from autism people. The independent components can be identified for both EEG and fMRI. The analysis is proceeded by constructing statistical features for both identified components. A classifier is developed by k-means on a 2D feature projection space, EEG and resting state fMRI 4D dataset. Eigen vectors are obtained from the independent components extracted from EEG and fMRI datasets from data warehouse. The eigen values are sorted in order where the highest elements are considered for analysis. These highest values are clustered using $\mathrm{k}$ means and the obtained classifier accuracy is found to be comparable achievement with the state of art classification algorithms.

The clinical classifier in discriminating patients with healthy controls is achieved after due warehousing and preprocessing of the acquired EEG and fMRI signals. The only remark to be considered is the processing of huge data as the 10 scans of fMRI and 10 subjects EEG signals need to be provided warehouse security, preprocessed, identify independent components and classification in projection space.

\section{CONCLUSION AND FUTURE SCOPE}

The classifier obtained by the fusion of features extracted from the independent components of EEG and fMRI are subjected to k means clustering on 2-D projection space. The results obtained using fMRI has advanced cognitive neuroscience research. An detailed attention on integration and paradigm analysis, a more prominent consideration on particular guess comprehend by commencing collection of data, and amplified utilization of thinking and arithmetic models to depict disguised process.

\section{REFERENCES}

1. Diagnostic Chen, Colleen P., et al. "Diagnostic classification of intrinsic functional connectivity highlights somatosensory, default mode, and visual regions in autism." NeuroImage: Clinical 8 (2015): 238-245.

2. De Martino, Federico, et al. "Classification of fMRI independent components using IC-fingerprints and support vector machine classifiers." Neuroimage 34.1 (2007): 177-194.

3. SamanSarraf, GhassemTofighi,'Classification of Alzheimer's Disease Using fMRI Data and Deep Learning Convolutional Neural Networks',2016.

4. Sarraf, Saman, and Ghassem Tofighi. "Classification of alzheimer's disease using fmri data and deep learning convolutional neural networks." arXiv preprint arXiv:1603.08631 (2016).

5. L. T. K. Vo, H. A. Sharma, Y. M. Wang, D. B. Walther, A. F. Kramer, and $\mathrm{W}$. Olivero, Classification technique to detect activation patterns in pain fMRI data, 2014

6. Allen, Elena A., et al. "A baseline for the multivariate comparison of resting-state networks." Frontiers in systems neuroscience 5 (2011): 2 .. .

7. Ashburner, John, and Karl J. Friston. "Unified segmentation." Neuroimage 26.3 (2005): 839-851.

8. Berger, Andrea, and M. I. Posner. "Pathologies of brain attentional networks." Neuroscience \& Biobehavioral Reviews 24.1 (2000): 3-5..

9. Richard Beare1 \& Chris Adamson1 \& Mark A. Bellgrove2 \& Veronika Vilgis \&Alasdair Vance3 \& Marc L. Seal \& Timothy J. Silk, "Altered Structural Connectivity In ADHD: A Network Based Analysis", Brain Imaging And Behavior. Springer Science and business Media, New York 2016.

10. K.Thaiyalnayaki, 'Identification and Research of Adhd and Healthy Controls using Fmri', International Journal of Engineering and Advanced Technology, Volume-8 Issue-6S, August 2019.

11. Mohankumar, S. "Analysis of different wavelets for brain image classification using support vector machine." International Journal of Advances in Signal and Image Sciences 2.1 (2016): 1-4.

12. Lebaka, Sivaprasad, Tammineni Sreelatha, and K. Venkatramana Reddy. "The Glaucoma Image Classification Using Texture Features For SVM Classifier." Indian Journal of Public Health Research \& Development $9.10(2018$ 
13. J.V. Kumar, and C.K.C. Paul, "Design Of Enhanced Sqrt Carry Select Adder For Vlsi Implementation Of 2d-Discrete Wavelet Transform". International Journal Of MC square scientific research, Vol. 9, No. 2, 2017, pp. 64-69.

\section{AUTHORS PROFILE}

Dr.K.Thaiyalnayaki currently working as Associate Professor in Saveetha School of Engineering,SIMATS is having 20 years of experience in teaching and Research in the field of Multi Dimensional Image Processing, Advanced signal Processing, Advanced Neural Network Deep Learning and VLSI Signal Processing. She has acquired $\mathrm{Ph} . \mathrm{D}$ and M.E specialized in Applied Electronics, Gold Medalist from College of Engineering, Guindy, Anna University, Chennai and U.G from Madurai Kamaraj University. She has 25 Research publications in reputed International Journals and conferences to her credit. 